EPJ Web of Conferences 114, 02002 (2016)

DOI: $10.1051 /$ epjconf/201611402002

(C) Owned by the authors, published by EDP Sciences, 2016

\title{
Experimental research of heterogeneous nuclei in superheated steam
}

\author{
Ondřej Bartoš ${ }^{1,2, a}$, Michal Kolovratník ${ }^{1}$, Bohuslav Šmíd ${ }^{2}$ and Jan Hrubý ${ }^{2}$ \\ ${ }^{1}$ Czech Technical University in Prague, Department of Energy Engineering, Technická 4, Prague 6, 166 07, Czech Republic \\ ${ }^{2}$ Institute of Thermomechanics of the CAS, v.v.i., Dolejškova 5, 18200 Prague 8, Czech Republic
}

\begin{abstract}
A mobile steam expansion chamber has been developed to investigate experimentally homogeneous and heterogeneous nucleation processes in steam, both in the laboratory and at power plants using the steam withdrawn from the steam turbine. The purpose of the device is to provide new insight into the physics of nonequilibrium wet steam formation, which is one of the factors limiting the efficiency and reliability of steam turbines. The expanded steam or a mixture of steam with a non-condensable gas rapidly expands in the expansion chamber. Due to adiabatic cooling, the temperature drops below the dew point of the steam at a given pressure. When reaching a sufficiently high supersaturation, droplets are nucleated. By tuning the supersaturation in the so-called nucleation pulse, particles of various size ranges can be activated. This fact is used in the present study to measure the aerosol particles present in the air. Homogeneous nucleation was negligible in this case. The experiment demonstrates the functionality of the device, data acquisition system and data evaluation methods.
\end{abstract}

\section{Introduction}

The increasing global demand for electric power on the one hand, and strict requirements for reducing the emissions of pollutants and other environmental impacts of power production on the other, presents a new challenge of enhancing the efficiency of energy conversion systems. In most western countries including the Czech Republic - most power is generated in nuclear and fossil-fuelled power plants. Because of the geographical conditions, other energy resources are limited and thermal power plants will continue their leading position in the coming decades as well. The requirement of efficiency and reliability enhancements also affects the steam turbine as an important part of the Rankine cycle of thermal power plants. In the lowpressure part (LP) of the steam turbine, the steam undergoes the process of nucleation and condensation into primary droplets [1]. Some of the primary droplets hit the blades and other surfaces and form a liquid film. Secondary droplets are formed by the disruption of the liquid film from blades and casing [2]. The nonequilibrium formation of the liquid phase significantly affects the budget of energy conversion efficiency. Despite the hundred-year development of steam turbines and investigations of the underlying physical processes, the microphysics of liquid phase formation is still only partially understood. Consequently, the mathematical models of liquid phase formation currently available to turbine designers and operators provide incomplete information on the effect of design and operation modifications on the efficiency and reliability of steam turbines. The rapid progress of computing hardware and computational fluid dynamics (CFD) allows for more and more accurate turbine-design computations. It has become a necessity to also bring the models of nonequilibrium condensation processes to a new level. The study of the effects of impurities in the super-heated steam is an important contribution for a better understanding of wet steam formation. The impurities in the super-heated steam are particularly important at the beginning of the nucleation and condensation processes. It can be expected that the impurities act as heterogeneous nucleation seeds.

\section{Heterogeneous nucleation}

Heterogeneous nucleation can be an important mechanism of early droplet formation (the so-called early condensate), because it requires a lower supersaturation to start $[3,4]$. The actual value of supersaturation at which a heterogeneous particle is activated depends on its size and wetting properties for insoluble particles, size and solubility for soluble particles. Also the solubleinsoluble transition has been considered. It is assumed that the origin of most particles present in steam is in the droplets carried over by the steam from the boiler. In the superheater, the droplets dry out and low-volatile compounds (typically simple salts and oxides) dissolved in the droplets form fine particles. Consequently, such particles would be completely or partly soluble. Until

\footnotetext{
${ }^{\mathrm{a}}$ Corresponding author: ondrej.bartos@fs.cvut.cz
} 
recently, no data existed on heterogeneous particles in steam. Pioneering work [5] by the present team showed $3 \times 108$ to $6 \times 109$ particles per kilogram of steam in various power plants. The size distribution was characterized by the Sauter diameter of $30 \mathrm{~nm}$. However, chemical analysis of condensed steam samples showed that only a small fraction of the low-volatile matter was captured by the present instrumentation, leading to the hypothesis that most of the matter is dispersed in a large number of particles smaller than $3 \mathrm{~nm}$ (the present detection limit).

\section{Expansion chamber}

The main motivation for the development of the expansion chamber was the lack of a suitable identification tool for identification of the particles size down to $10 \mu \mathrm{m}$. The classical aerosol devices like a condensing particle counter (CPC) has a detection limit of $3 \mathrm{~nm}$ but the efficiency of the detection for small particles are low. The application of a scanning mobility particle sizer (SMPS) is difficult due to the insufficient number density of the particles and a high deposition rate in the sampling tubes [5]. The developed expansion chamber provides a new approach to particle identification. The measurement is performed as a series of expansions with different back pressures. The expansion ratio (ratio of the final to initial pressures) $R$ and saturation $\mathrm{S}$ change together with the expansion and this means that different groups of heterogeneous particles are involved in the nucleation and condensation processes. In case heterogeneous particles are present in the sample, it can be expected that particles with a radius bigger than the critical droplet radius $\mathrm{r}^{*}$ will grow and ultimately become droplets of a diameter on the order of $100-1000 \mathrm{~nm}$, which can be detected optically. The critical radius can be estimated as

$$
r^{*}=\frac{2 \sigma}{\rho_{l} r T_{g} \ln S}
$$

Here, $\sigma$ is surface tension, $\rho l$ is droplet density, $r$ is the specific gas constant and $\mathrm{Tg}$ is the temperature of the gas, and $\mathrm{S}$ is supersaturation. Because the present experiments are performed at low pressures, the supersaturation can be computed as a ratio of the actual vapour pressure pv and the saturation vapour pressure ps corresponding to the actual temperature,

$$
S=\frac{p_{v}}{p_{s}}
$$

The purpose of the expansion chamber is to carry out the expansion of the sampled steam from the steam turbine in order to study homogeneous and heterogeneous nucleation processes. The entire device is mobile so that measurements can be performed on site at a power station. Usually the steam from the feed water heater or extraction pipes is used.

During its transport in the connecting tubing and in the expansion chamber, the diluted sample is kept at a constant temperature sufficiently high to avoid any condensation. A schema of the chamber is provided in Fig. 1.

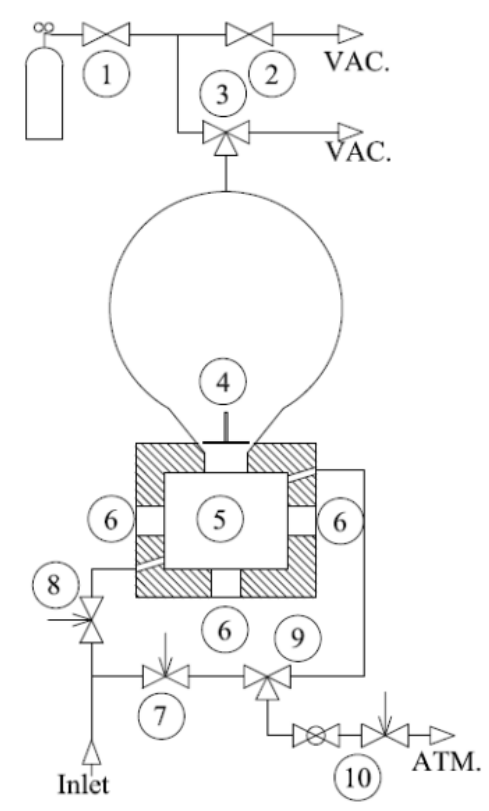

Figure 1. 1. High pressure valve, 2. Low pressure valve, 3. Evacuation valve, 4. Expansion valve, 5. Test section, 6. Optical window, 7. Bypass, 8. Chamber valve, 9. Bypass switching valve, 10. Outflow valves. Description is given in the text.

Solenoid valves 1 and 2 are switched by a PID controller to maintain the desired back pressure. Before the expansion, test section 5 of the chamber is flushed with the sample for a sufficient time to achieve adsorption equilibrium on the walls of the chamber and tubing. The specially designed fast-opening expansion valve 4 is actuated electro-pneumatically. Within a few milliseconds (see Fig. 2 showing the pressure trace), the vapour-gas mixture expands from the initial chamber pressure to the adjusted back pressure. During the expansion, the sample flow passes through the bypass with valve 7 to avoid pressure rise in the sampling tubing, valve 8 is closed. This valve presents a similar pressure drop to that of the test section and expansion valve. The 3 -way valve 9 switches the sample flow from bypass or the test section. Throttling valve 10 is used to adjust the desired flow rate through the device. The laser light scattering and extinction measurements are performed through windows 6 .

\section{Methods}

Special data acquisition and evaluation codes have been developed. The entire data processing procedure is not straightforward and requires several particular steps. The measurement sequence is triggered by the pressure drop in the chamber. The rapid pressure decrease is measured by a piezoelectric transducer with a Kistler charge amplifier. The temperature of the entire expansion chamber and the expansion valve is maintained at a constant value by a circulating bath thermostat. Together with the pressure trace, optical signals are also acquired: the power of the laser beam attenuated by the cloud of 
nucleated droplets is measured by a photodiode, the power of the incident beam is measured by another photodiode, the intensity of the scattered light measured with a photomultiplier.

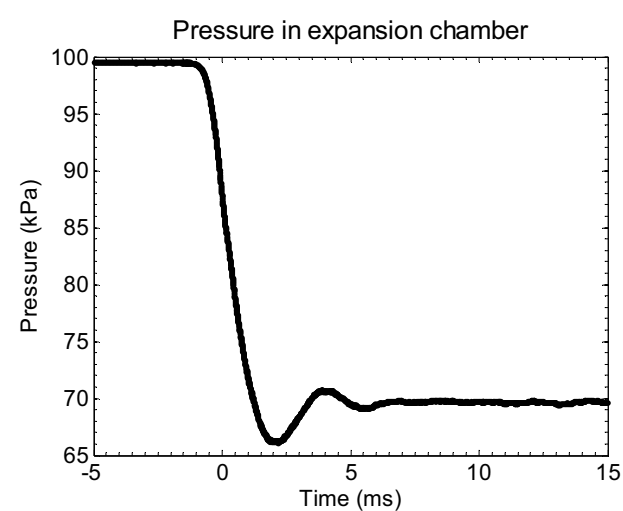

Figure 2. Pressure profile during the expansion.

Light extinction for determining the particle number density and the light scattering at 90 degrees for determining the droplets growth is acquired as a function of time. The determination of the number density of the droplets is based on the Lambert Beer law. The extinction ratio $\mathrm{I} / \mathrm{Io}=\mathrm{f}(\lambda)$ is measured as a function of time. Applying the Mie theory of light scattering on water droplets one can find the size distribution of the droplets $\varphi(D)$ and number density. In general a system of illconditioned equations is obtained [6]:

$$
\frac{1}{\ell} \ln \left(\frac{I_{o}}{I}\right)_{i}=\frac{\pi}{4} N_{v} \int_{0}^{D_{\infty}} \mathrm{Q}\left(\pi D / \lambda_{i}\right) \cdot \varphi(D) \cdot D^{2} \mathrm{~d} D .
$$

Here, $\ell$ is the length of the measurement section, $\mathrm{Nv}$ is the volumetric number density of droplets, $\mathrm{Q}(\pi \mathrm{D} / \lambda \mathrm{i})$ is the extinction coefficient according to the Mie theory, D droplet diameter, $\varphi(D)$ size distribution function of the droplets and $\mathrm{i}=1,2, \ldots k$ is the chosen number of the particular wavelengths $\lambda_{1,2,3 \ldots k}$ and this presents the number of the equation in the system.

Almost all droplets are nucleated within the so-called nucleation pulse, i.e. the pressure dip shown in Fig. 2. Consequently, the cloud of droplets can be considered as monodisperse and a monochromatic $(675 \mathrm{~nm})$ laser is used as the light source. Hence, equation (3) reduces simple form (4),

$$
\frac{I}{I_{0}}=\exp \left(-\pi N_{v} D \lambda Q L\right)
$$

The light scattering intensity as the function of the droplet radius is shown in Fig. 3.

The comparison of the peeks position or in general the theoretical and measured light intensity profile one can reach a dependence on droplets growth over time. Nevertheless, the desired result is the size distribution function of the heterogeneous seeds in the steam/gas at the moment the profile of the water droplet is determined. The application of this knowledge and the theory of droplet growth can help to achieve the final distribution function of the impurities in the gas.

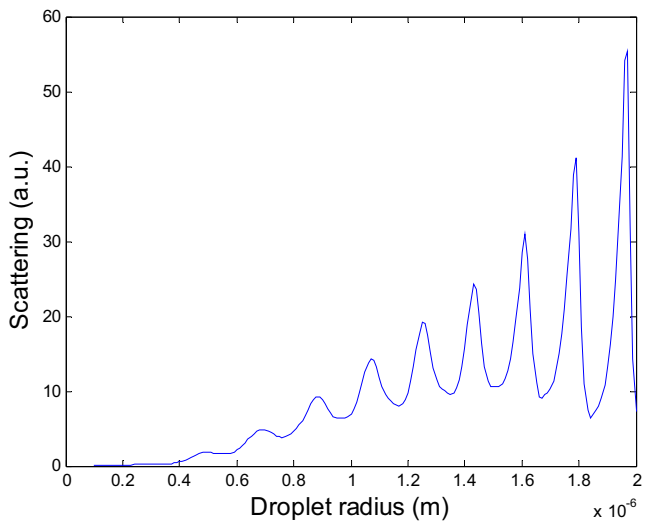

Figure 3. Dependence of the light intensity for light scattering at $90^{\circ}$ for wavelength $675 \mathrm{~nm}$ and variable droplet radius.
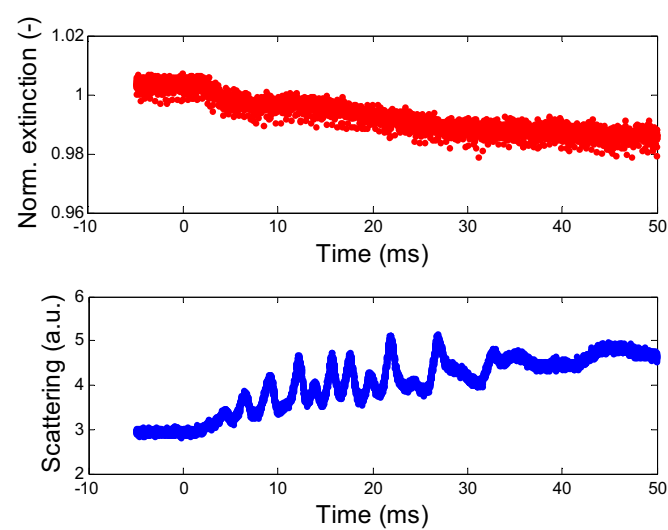

Figure 4. Profile of the light extinction and light scattering at $90^{\circ}$.

The growth of the droplet which start condensing on the foreign particle can be written with respect to some simplification $[7,8]$

$$
r=\left(r_{*}^{2}+\frac{2(s-1)}{C_{1}+C_{2}}\left(t-t_{0}\right)\right)^{0.5}
$$

where $\mathrm{S}$ is the saturation of the steam, $\mathrm{r}^{*}$ is the critical diameter or minimum size of the active foreign particle involved in the condensation. $\mathrm{C} 1$ and $\mathrm{C} 2$ are constants which respect diffusion of the water vapour in the gas, heat conductivity and physical properties of the gas and vapour.

Satisfactory results require a strong correlation between the measured diameters and the theoretical function of the droplet growth on the foreign particle over time. The whole data processing technique is sensitive to many variables and requires careful measurement.

\section{Results}

In this paper, an example of the results achieved by the measurement with the ambient air is presented. This kind of test proved useful because experiments at power plants are dependent on the operator's permission and can be performed on rare occasions. The unveiled test with the air was performed in the CTU laboratory. The measurement was used to evaluate the entire data acquisition and data processing method. The knowledge 
gained was used to enhance the measurement procedure. For example the fine adjustment of the control valves for maintaining the back pressure in the expanding vessel.

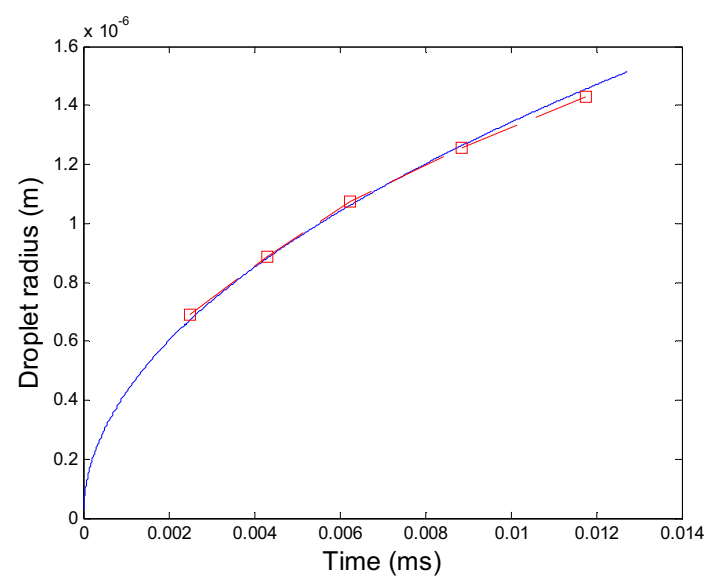

Figure 5. Example of the theoretical profile of the droplet growth compared with the measured values.

Fig.5 shows an example of the theoretical growth of the droplet over time together with the measured droplet size determined from the analysis of the light scattering. The expansion rate for the presented measurements was in the range $250 \div 350 \mathrm{~s}-1$ and the temperature of the gas was $20^{\circ} \mathrm{C}$. The relative air humidity was $53 \%$. Homogeneous nucleation was taken into account $[9,10]$ but for all measurements was considered as negligible because the saturation ratio (eq.5) never exceeded $S=3$ for all the expansions.

Measurement evaluations defined the requirements for precise control of the input parameters of the gas mixture. Precise modelling of the droplet growth and a model of the entire expansion process is necessary for determining the minimum starting diameter of the heterogeneous seeds in the gas mixture. In Fig. 6 the right part of the size distribution function is presented for the ambient air. The data correlates with that found in the literature [11].

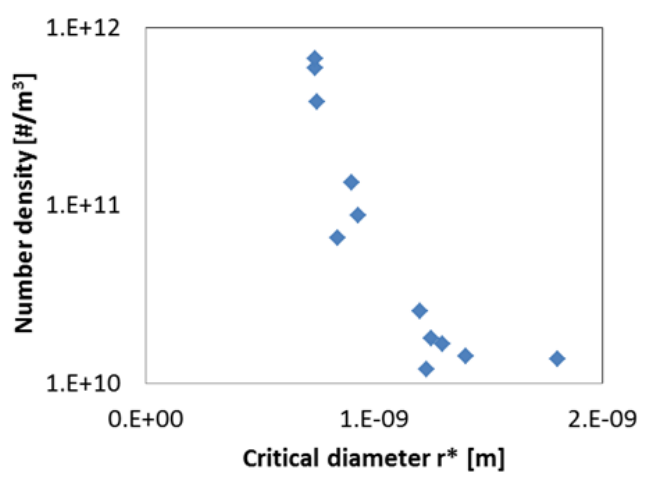

Figure 6. The determined heterogeneous particles size distribution function.

\section{Conclusions}

The evaluation measurement proves that the expansion chamber is a sufficient tool for the identification of foreign impurities in steam. Nevertheless, the entire process of the evaluation of the heterogeneous seeds size distribution function is very sensitive and requires a deep understanding of the nucleation process and precise measurement. Further enhancement of the determination method will focus on detailed knowledge of the input gas mixture properties and modelling of the expansion process. Other efforts will be focused on earlier detection of the growing particles through the use of light with a shorter wave length and by adjusting the entire optical system.

\section{Acknowledgements}

We gratefully acknowledge the support by Grant No. 101/11/1593 of the Grant Agency of the Czech Republic and by the Grant Agency of the Czech Technical University in Prague, grant No. SGS 14/183.

\section{References}

1. V. Petr, M. Kolovratník, Wet steam energy loss and related Baumann rule in low pressure steam turbine. Proceedings of the Institution of Mechanical Engineers Part A - Journal of Power and Energy. 2014, vol. 228, no. 2, p. 206-215. ISSN 0957-6509.

2. O. Bartoš, X. Cai, M. Kolovratník, EPJ Web of Conferences, 2014, vol. 67, ISSN 2100-014X.

3. W.T. Lindsay, Behavior of impurities in steam turbines, Power engineering, Vol. 5, pp. 68-72, 1975.

4. W.G. Steltz, et al, The verification of concentrated impurities in low-pressure steam turbines, TASME, J. of engineering for power, Vol. 105, pp. 192-198. 1983.

5. M. Kolovratník, J. Hrubý,V. Ždímal, O. Bartoš, I. Jiříček et al.: Nanoparticles found in superheated steam - A quantitative analysis of possible heterogeneous condensation nuclei. Proceedings of the Institution of Mechanical Engineers Part A Journal of Power and Energy. 2014, vol. 228, no. 2, p. 186-193. ISSN 0957-6509.

6. C.F. Bohren, D.R. Huffman, Absorption and Scattering of Light by Small Particles. John Wiley \& Sons, Inc., USA, 1998

7. H. Köhler, The nucleus in and the growth of hygroscopic droplets. Trans. Faraday Soc. 32 (1936) $1152-1161$

8. W. E. Howell, The growth of cloud drops in uniformly cooled air. J. Meteor., 6, 134-149,1949.

9. V. Holten, Water nucleation. PhD Thesis, Eindoven: Eindhoven University of Technology 2009

10. J.Wolk, at al. Empirical function for homogeneous water nucleation rates J.Chem.Phys.117,4954(2002)

11. W.C. Hinds, Aerosol technology (Second Ed.). Wiley, New York, 1999. 